\title{
Improving Quantitative Understanding Using Spreadsheet Models
}

Donovan, Therese M., and Charles W. Welden. 2002. Spreadsheet exercises in ecology and evolution. Sinauer, Sunderland, Massachusetts. xi +556 p. $\$ 24.95$, ISBN: 087893-156-2.

Donovan, Therese M., and Charles W. Welden. 2002. Spreadsheet exercises in conservation biology and landscape ecology. Sinauer, Sunderland, Massachusetts. xi +464 p. \$24.95, ISBN: 0-87893-159-7.

I am a spreadsheet dummy. It was with a lot of trepidation that I agreed to review a new pair of books by Therese M. Donovan and Charles W. Welden entitled Spreadsheet exercises in ecology and evolution, and Spreadsheet exercises in conservation biology and landscape ecology. After all, it had been less than a year since I learned how to calculate average values for a column of data. Always in search of interesting activities for my sophomore General Ecology class, I agreed to take a look at the books. I figured if I could learn how to do these exercises, so could my students. Boy, am I ever glad I tried!

The books are meant to supplement the primary text in undergraduate and beginning graduate courses in ecology. Each chapter presents a spreadsheet activity designed to reinforce basic concepts in ecology, evolution, landscape ecology, conservation, and statistics. All chapters have a similar format: a statement of objectives; an introduction with enough information for the reader to (1) understand what information the analysis can provide, (2) relate the analysis to basic concepts, and (3) understand the assumptions made by the analysis for better subsequent interpretation; an annotated set of instructions designed for use with Microsoft Excel ${ }^{\text {(ii }}$; questions designed to make the reader interpret data generated; and literature for additional reading. No software is provided-each model and formula are generated by the student, enabling them to learn spreadsheet software, as well as understand relationships among parameters. One benefit for me was better learning how to use spreadsheets. I have requested that all of my graduate students and research undergraduates complete the first five exercises.

There is a considerable amount (30-40\%) of overlap in the content of the two texts. Forty-three exercises are presented in Spreadsheet exercises in ecology and evolution; 36 exercises are presented in Spreadsheet exercises in conservation biology and landscape ecology. Topics are organized into three sections in each text. The section on "Spreadsheets and statistics" found in both books provides excellent introduction to the use of spreadsheet functions and macros, statistical distributions, and hypothesis testing. These would be of use in any ecology, environmental science, or beginning biometry course. I began my exploration of spreadsheet modeling here, completing the exercises on "Mathematical functions and graphs," "Spreadsheet functions and macros," "Statistical distributions," and "Hypothesis testing: alpha, beta, and power." The instructions make completing the exercises fairly easy in Microsoft Excel ${ }^{\text {, }}$, though I had some frustrating moments translating them to QuattroPro ${ }^{\circledR}$, which is often my preferred software. These exercises are a perfect way to introduce students to the utility of spreadsheets. The content in "Statistical distributions" and "Hypothesis testing" provides an excellent introduction to normal, frequency, and Poisson distributions, Type I and Type II errors, and $t$-tests. The statistical analyses do not go beyond these concepts. My only criticism here is that commonly used tests in ecology, including non-parametric and multivariate statistic, are not mentioned. For this reason, the exercises are somewhat limited to more introductory level ecology courses.

Both texts provide excellent exercises to help students understand population growth and dynamics. The exercises I tried included "Geometric and exponential population model," "Island biogeography," "Life tables, survivorship curves, and population growth," and "Source-sink dynamics." Each would help students understand the mathematics behind graphics presented on these topics in most ecology texts. The exercises varied in their complexity and in the amount of time that is required to complete them. The chapter 
"Geometric and exponential population model" should be fairly easy for an introductory ecology student to complete, even in courses where the calculus is not explained in depth. At the same time, this chapter provides a nice discussion of the two models and would help reinforce understanding of how demographic processes affect growth, assumptions of the two models, and relationships between them. The "Island biogeography" and "Source-sink" chapters take more time to complete, yet show the effects of immigration and extinction on population growth. One nice aspect about the organization of the exercises is that they are divided into sections of varying complexity. Instructors could choose to use only some sections as appropriate for their course; however, the exercises in this suite build nicely on each other and as a group provide a lot of opportunity for students to improve their quantitative understanding of population growth.

Interesting chapters unique to the book Spreadsheet exercises in ecology and evolution include "Evolutionarily stable strategies and group versus individual selection" which includes a model of simple game theory; "Niche breadth and resource partitioning"; "Quantitative genetics"; and "Heritability."

A number of chapters in Spreadsheet exercises in conservation biology and landscape ecology overlap with those in the ecology and evolution text. There are some important differences however that deal with landscape ecology. One excellent exercise is the chapter "VBA landscape programming" which describes how to use Visual Basic for Applications as a tool along with the spreadsheet. This exercise allows the student to design a raster-based landscape. Tools used in this chapter are used in a subsequent chapter called "Neutral landscape theory and connectivity." These exercises took quite some time to complete and would be rec- ommended as a lab activity or mini-project. They are not appropriate as a short homework assignment.

Internet-based support is available for students and instructors through the publisher's website at www.sinauer.com. Instructors may access spreadsheet templates as well as completed spreadsheets and answers to questions. When I reviewed the sites they were still under construction and not all exercise templates were on the site. The information available at that time would be of use to instructors and students alike. The completed spreadsheets are password protected, while the answers to questions are not, meaning students have access to them.

Overall these texts should be welcome on any ecology instructor's bookshelves. At less than $\$ 25.00$ they are a bargain. Because there is so much overlap in content, one or the other should be chosen based on course needs and personal interest. For courses with more of an evolutionary emphasis, Spreadsheet exercises in ecology and evolution would be most appropriate. Spreadsheet exercises in conservation biology and landscape ecology should be used in courses emphasizing natural resources management, reserve design, and landscape ecology. For me the texts provided a long-overdue opportunity to learn how to effectively use spreadsheets for data analysis and modeling. Finally, these volumes should motivate instructors to improve their students' quantitative understanding of ecological and evolutionary concepts.

\section{Michelle A. BAKer}

Utah State University

Department of Biology

Logan, Utah 84322

E-mail: mbaker@biology.usu.edu 